\title{
Editorial
}

\section{The Accessory Navicular in Children and Adolescents: What We know and What We can do}

The navicular, also known as os naviculare or the tarsal scaphoid, derives its name from Latin "navis" or ship. It is interposed between the head of the talus and the cuneiforms, and it forms an important part of the medial longitudinal arch of the foot. The navicular tuberosity, a bony prominence on the medial end of the bone, provides insertion to the tibialis posterior tendon, which is the most important dynamic stabilizer of the medial longitudinal arch of foot. ${ }^{1}$

Accessory ossicles are common occurrences in the skeleton. In a radiographic study in Turkish subjects, the accessory navicular (AN) was found to be the most common ossicle of the foot. ${ }^{2}$

The navicular is the last tarsal bone to ossify, and it has a single center of ossification, which appears around 2.7 to 4 years of age. The AN usually develops due to a secondary ossification center ${ }^{1}$ and can be classified into three types. ${ }^{1}$ Type $1 \mathrm{AN}$ is generally 2 to $3 \mathrm{~mm}$ in diameter, round to oval in shape, and a small sesamoid contained within the tendon of the tibialis posterior, on its plantar aspect, near the calcaneonavicular ligament. It is rarely symptomatic and accounts for a third of all ANs.
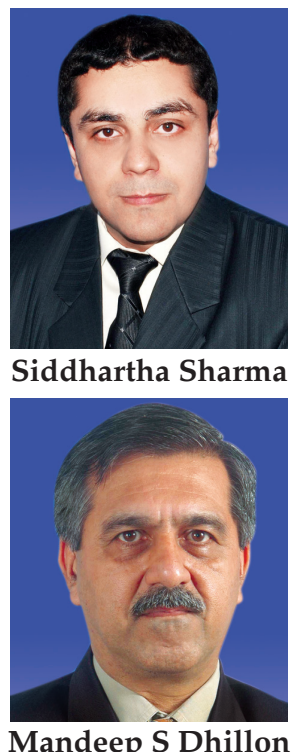

Type 2 AN is larger (8-12 $\mathrm{mm})$ in size, triangular in shape, and has a synchondrosis with the main navicular bone. This type is often symptomatic and is further subdivided into two types based on its connection to the main bone. Type 2a AN is connected to the navicular at a less acute angle and is susceptible to tension forces, which may result in avulsion. Type $2 \mathrm{~b}$ AN is connected to the navicular at a more acute angle and is susceptible to shearing forces.

Type $3 \mathrm{AN}$ is connected to the navicular by a bony bridge, and like type $1 \mathrm{AN}$, it is rarely symptomatic. The most common clinical presentation of AN is pain localized to the medial border of foot, which worsens with activity and weight bearing. Although many cases of AN are associated with pes planus, a causal relationship has not been established so far. A recent article published by Park et $\mathrm{al}^{3}$ showed that the calcaneal pitch angle in AN was significantly smaller as compared with controls; the naviculocuboid overlap, talonavicular coverage angle, the lateral talo-first metatarsal angle, and the anteroposterior talo-first metatarsal angle were significantly larger in the AN group. However, the degree of flatfoot was not associated with the occurrence or severity of symptoms in AN. ${ }^{3}$

In addition to pain, many patients with AN report discomfort with narrow footwear. A thorough history and physical examination are mandatory to rule out other causes of medial foot pain.

The most important imaging investigation for AN is plain radiography. The AN can often be identified on plain radiographs of the foot, but is best delineated on $45^{\circ}$ external oblique view of the foot. Standing anteroposterior and lateral radiographs of both feet are important to assess for pes planus. Magnetic resonance imaging (MRI) and bone scans have been used to differentiate AN from a fracture of the navicular tuberosity. A tuberosity fracture usually presents with sharp edges on plain radiographs, which is in contrast to smooth borders in AN. Technetium bone scan shows increased uptake and MRI reveals the presence of bone edema in a tuberosity fracture. ${ }^{1}$

The treatment of AN is primarily nonoperative. Activity restriction and modification along with shoe-wear modifications and orthoses can help relieve symptoms in a number of cases. Wider shoes with orthoses that offload the midfoot should be prescribed. A trial of casting, which works by preventing the pull of tibialis posterior, may be necessary to alleviate the symptoms. Corticosteroid injections may be used to decrease pain; however, care must be taken not to inject directly into the tendon substance as this may lead to rupture. Ultrasonography or image intensification radiology may be used to guide corticosteroid injection. ${ }^{1}$

Surgery is indicated for symptomatic AN, which has not responded well to nonoperative methods. The different surgical techniques include excision of the AN, Kidner procedure (excision with advancement of the tibialis posterior tendon), percutaneous drilling, and arthrodesis of the AN.

Excision may be a reasonable option for a small AN along with a normal native navicular and no planovalgus deformity. In such cases, the AN can be excised without disturbing the continuity of the tibialis posterior tendon. ${ }^{4}$ In most cases of type 2 and type $3 \mathrm{AN}$, the tibialis posterior tendon is noted to have an abnormal insertion on to the AN. In such cases, the Kidner procedure is preferable. This consists of excision of the ossicle and rerouting of the tibialis posterior tendon into a more plantar position, thereby improving line of pull of this tendon. ${ }^{5}$ 
Nakayama et $\mathrm{al}^{6}$ have described percutaneous drilling technique for symptomatic type 2 ANs. The authors introduce a $1 \mathrm{~mm}$ Kirschner wire percutaneously from the AN, passing through the synchondrosis into the native navicular. This is thought to bring about fusion of the AN with the native navicular. Although excellent results were obtained in $96.8 \%$ of the cases, bony union was noted in $58 \%$ cases only. Therefore, more studies are needed before this method can be recommended for routine use. Arthrodesis of the AN with the native navicular (the modified Kidner procedure) has also been described. The synchondrosis is excised and the AN is fused to native using 2.7or 3.5-mm lag screws. However, this is possible only if the AN is large enough in size to accommodate a screw. The tibialis posterior tendon can be left attached to the AN. ${ }^{7}$ Adjunctive corrective procedures for concomitant pes planus may be needed in some cases.

To sum up, an accurate clinical and radiological evaluation is the key for managing AN. A vast majority of patients may be managed by nonoperative means, while surgery is indicated for uncommon refractory cases. Excision may be preferable for small AN; Kidner procedure is preferable for most symptomatic types 2 and 3 ANs. In these cases, arthrodesis of the AN is also a reasonable alternative if the AN is sufficiently large in size.

\section{REFERENCES}

1. Leonard ZC, Fortin PT. Adolescent accessory navicular. Foot Ankle Clin 2010 Jun;15(2):337-347.

2. Coskun N, Yuksel M, Cevener M, Arican RY, Ozdemir H, Bircan O, Sindel T, Ilgi S, Sindel M. Incidence of accessory ossicles and sesamoid bones in the feet: a radiographical study of the Turkish subjects. Surg Radiol Anat 2009 Jan;31(1):19-24.

3. Park H, Hwang JH, Seo JO, Kim HW. The relationship between accessory navicular and flat foot: a radiologic study. J Pediatr Orthop 2015 Oct-Nov;35(7):739-745.

4. Grogan D, Gasser S, Ogden J. The painful accessory navicular: a clinical and histopathological study. Foot Ankle 1989 Dec;10(3):164169.

5. Kidner FC. The pre-hallux (accessory scaphoid) in its relation to the flat-foot. J Bone Joint Surg Am 1929 Oct;11(4):831-837.

6. Nakayama S, Sugimoto K, Takakura Y, Tanaka Y, Kasanami R. Percutaneous drilling of symptomatic accessory navicular in young athletes. Am J Sports Med 2005 Apr;33(4):531-535.

7. Malicky ES, Levine DS, Sangeorzan BJ. Modification of the Kidner procedure with fusion of the primary and accessory navicular bones. Foot Ankle Int 1999 Jan;20(1):53-54.

Siddhartha Sharma

Assistant Professor

Department of Orthopaedics

Postgraduate Institute of Medical Education and Research Chandigarh, India

Mandeep S Dhillon

Editor-in-Chief

Journal of Foot and Ankle Surgery

Professor and Head

Department of Orthopaedics

Postgraduate Institute of Medical Education and Research

Chandigarh, India 